\title{
EL MAL DE LA PIEDRA
}

\author{
Victoria Gonzalo Rodríguez, Mariano Pérez Albacete' y Enrique Pérez-Castro Ellendt².
}

Servicio de Urología. Hospital General Yagüe. Burgos. España.

'Servicio de Urología. Virgen de la Arrixaca. Murcia. España.

¿Unidad de Urología. Clínica La Luz. Madrid. España.

Resumen.- OBJETIVO: En este trabajo se realiza una descripción histórica de los descubrimientos sobre la litiasis y la evolución de su tratamiento. Se exponen los antecedentes que llevan al nacimiento de la endourología y de la litotricia por ondas de choque.

MÉTODOS: Hemos revisado los libros y las publicaciones de la historia de la urología y de la endourología.

RESULTADOS/CONCLUSIONES: Conocer la historia y el tratamiento de la litiasis, las aportaciones de los urólogos españoles así como el desarrollo de los materiales que han contribuido a conseguirlo.

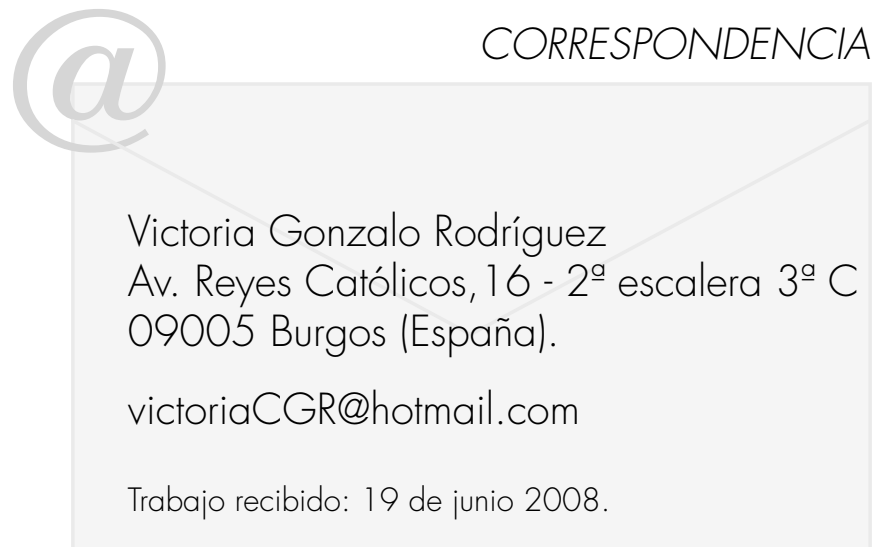

Palabras clave: Historia. Talla vesical. Endourología. Litotricia.

Summary.- OBJECTIVES: In this paper we describe the history of lithiasis and the development of its treatment. We discuss the steps that led to the birth of endoscopic surgery and extracorporeal shockwave lithotripsy.

METHODS: We reviewed books and writings of History of Urology and Endourology.

RESULTS/CONCLUSIONS: To know a little of the history and treatment of lithiasis, the contributions of Spanish urologists, as well as the instruments that made it possible.

Keywords: History. (Cystostomy) Bladder incision. Endoscopic Surgery. Lithotripsy.

\section{INTRODUCCIÓN}

La historia de la urolitiasis es posiblemente tan vieja como la de la humanidad. El primer cálculo vesical (1-3) fue descubierto 1901 por Elliot Smith en una tumba prehistórica (4800 a. de C.) Por su parte Shattock describió la existencia de un cálculo junto a la columna vertebral de una momia de la segunda dinastía (3000 años a. de C.) y que probablemente sea el cálculo renal más antiguo que se conoce.

Hipócrates en el año 460 a. de C. describe la semiologia del cólico renal y sus teorías sobre la litogénesis (Figura 1).

Posteriormente hay un periodo de más de tres siglos sin ningún descubrimiento importante en éste campo hasta llegar a Celso que describió la téc- 
nica de la Litotomía, consistente en una talla perineal, dada la sencillez de la técnica y el empleo escaso de material esta técnica fue denominada "apparatus minor." A pesar de ello la mitad de los pacientes morían como consecuencia de la hemorragia o la infección.

Años más tarde Galeno (Figura 2) perfecciona los conocimientos anatómicos de Celso.

A partir del siglo VII, después de la caída del Imperio Romano, la cirugía como otras ramas del saber, pasa a manos de los árabes, destacando Rhazes que formula la teoría de la formación de cálculos por exceso de sales en la orina.

En el siglo $X$, destacamos la figura del cirujano Abulcasis (Figura 3). Nacido en el año 936 en Zahara, el barrio real Córdoba. Posteriormente se traslada a Bagdad para completar sus conocimientos en medicina. La fama le llegó tras escribir una vasta enciclopedia médica, en treinta volúmenes o tratados conocida como "El Tesrif", donde hace aportaciones creando instrumental y procedimientos quirúrgicos, adjudicándole la paternidad de la cirugía moderna. Realiza una litotricia endoscópica "ciega", mediante la introducción en la vejiga de un aparato denominado "mashaba rebilia" que fragmentaba el cálculo

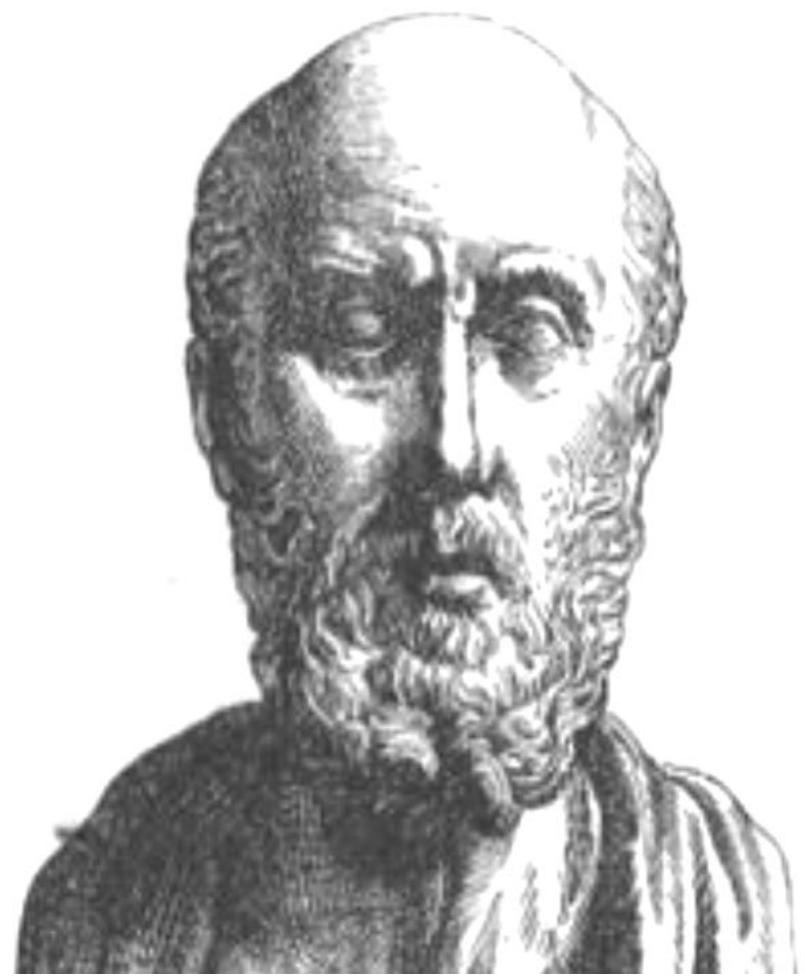

FIGURA 1. Hipócrates, describe la semiología del cólico renal. en el interior de la vejiga. Además fue uno de los primeros en realizar la talla vesical en mujeres. Estas técnicas suponen una anticipación de casi diez siglos a la reciente litotricia.

En Occidente durante un largo periodo que va desde el siglo VII al XV la cirugía es practicada por laicos a menudo sin conocimientos anatómicos adecuados.

En 1520 la técnica de la litotomía de Celso fue perfeccionada empleándose más instrumentación por lo que pasa a denominarse "apparatus major".

Pierre Franco describe la técnica de la talla suprapúbica (Figura 4) para la extracción de cálculos de gran tamaño que no podían extraerse mediante una talla perineal. Sin embargo está técnica no se difunde hasta bien avanzado el siglo XIX.

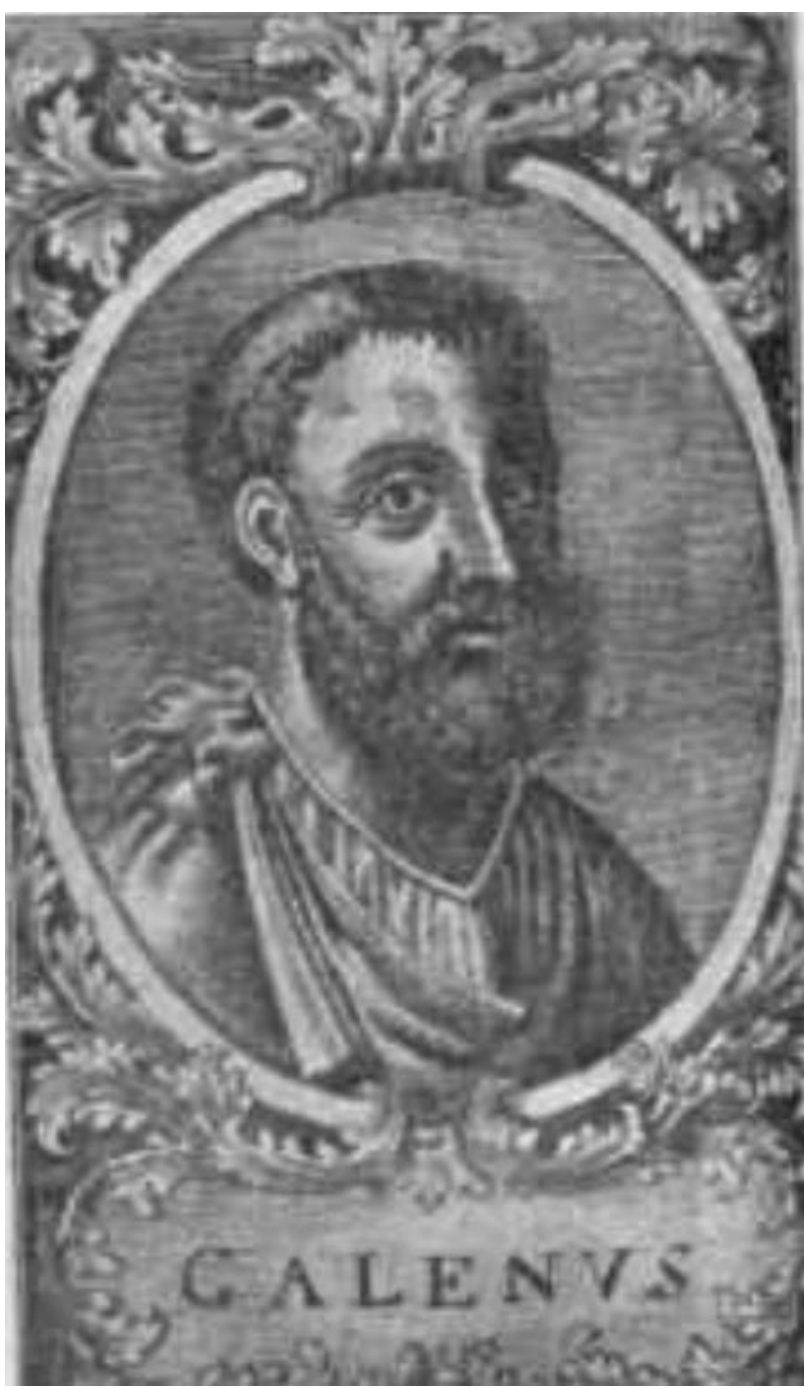

FIGURA 2. Galeno. 
En la misma época, Francisco Díaz nacido en Alcalá de Henares (Figuras 5 y 6), publica en 1588, el Tratado de las enfermedades de los riñones, vejiga y carnosidades de la verga y orina, con un capítulo dedicado a la formación de los cálculos, y realizando la primera descripción de la técnica de uretrotomía interna y cervicotomía. En este tratado reúne todo el saber urológico de la época.

Paralelamente a la cirugía del siglo XVIII se desarrollan diversos intentos de disolución de los cálculos mediante el empleo de sales alcalinas, carbonato potásico...etc. Se define la hipercalciuria como factor litogénico y se establece la importancia de la dieta en la litiasis úrica.

La era de la endoscopia comienza con el primer intento de examen de la cavidad vesical que realiza Philip Bozzini en Frankfurt on Main, en 1806. El instrumental esta formado por un tubo plateado que reflejaba la luz de una candela (lichtleiter).

En 1817 Jean Civiale, gran urólogo francés, no habiendo acabado la carrera de medicina, realiza intentos de destruir las litiasis sin dañar las paredes de la vejiga, para que el propio paciente expulse los fragmentos con la micción, llevando a cabo la primera litotricia transuretral en 1823. Para ello pasa un tubo a través de la uretra y pulveriza las litiasis con taladros o fresas, y sin saberlo inició lo que ahora denominamos cirugía mínimamente invasiva. En 1842 publica en París "Tratado de las Vías Urinarias". La Academia de las Ciencia de París comisionó al Dr
Jean Civiale a que realizará un soporte estadístico. La trascendencia de este trabajo radica es que constituye la primera investigación como "medicina basada en evidencias".

En 1828, Segalas $(4,5)$ presenta a la Academia de Medicina de Paris lo que denominó "especulo uretrocístico" para la exploración de la uretra, la vejiga, la vagina y el recto.

En 1853 Antonin Desormeaux describe otro instrumento " $L$ ' endoscope". Estaba constituido por un tubo único de visión con luz derivada del alcohol y con una lámpara de turpentina reflejada por un lente cóncavo con un sistema de iluminación superior para el estudio vesical que relata en el libro "Tratado de Endoscopia Urinaria y de las Afecciones Uretrovesicales" introduciendo el término Endoscopia, del griego "endo" (dentro) y "skopein" (examinar). En 1869 extirpa con este instrumental un pólipo uretral.

Llega el año 1879, y Max Nitze, médico alemán crea el cistoscopio verdadero, añadiendo al tubo óptico la luz producida por la lámpara incandescente, que permite la introducción de la luz directamente en la cavidad, ideada años antes por Edison.

En 1887 Albarran (4) le añade una pequeña uña para dirigir las sondas hacia los uréteres con precisión. Ringle modifica el sistema óptico de Nitze y más tarde Hirchovitz idea las fibras de cristal.

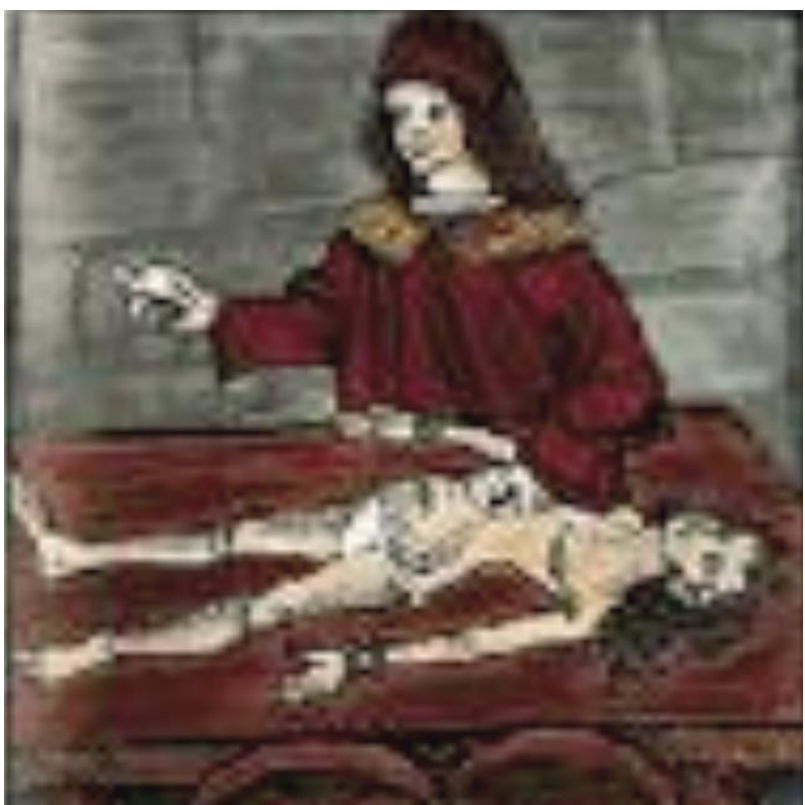

FIGURA 4. Litotomía 
Y lo que comenzó siendo exploratorio y por tanto diagnóstico se intentará pronto convertirlo en operativo. Así en 1931 Maximiliam Stern describe el resectoscopio y en 1932 Joseph Mc Carthy presenta un nuevo modelo. En 1939 Reed Nesbit desarrolló un aparato de resección prostática que era una modificación del instrumento de Mc Carthy que facilitaba al operador poder realizar la cirugía con una sola mano.

En 1948 durante su cautiverio en la cárcel de Fidel Castro José Iglesias desarrolla su "fleje", un mecanismo en el elemento de trabajo del resectoscopio que permitía utilizarlo con una sola mano y poder emplear lavado de doble corriente. Cuando sale de la cárcel emigra a USA donde lo patenta como profesor en el Martland Hospital de New Jersey. Este resectoscopio de succión continua revolucionó la cirugía endourológica al disminuir con dicha modificación el síndrome de resección transuretral de próstata.

En 1956 Harold Hopkins profesor de Óptica Técnica en la Universidad Inglesa de Reading desarrolla los "Hopkins Rod Lens" patente que le compró

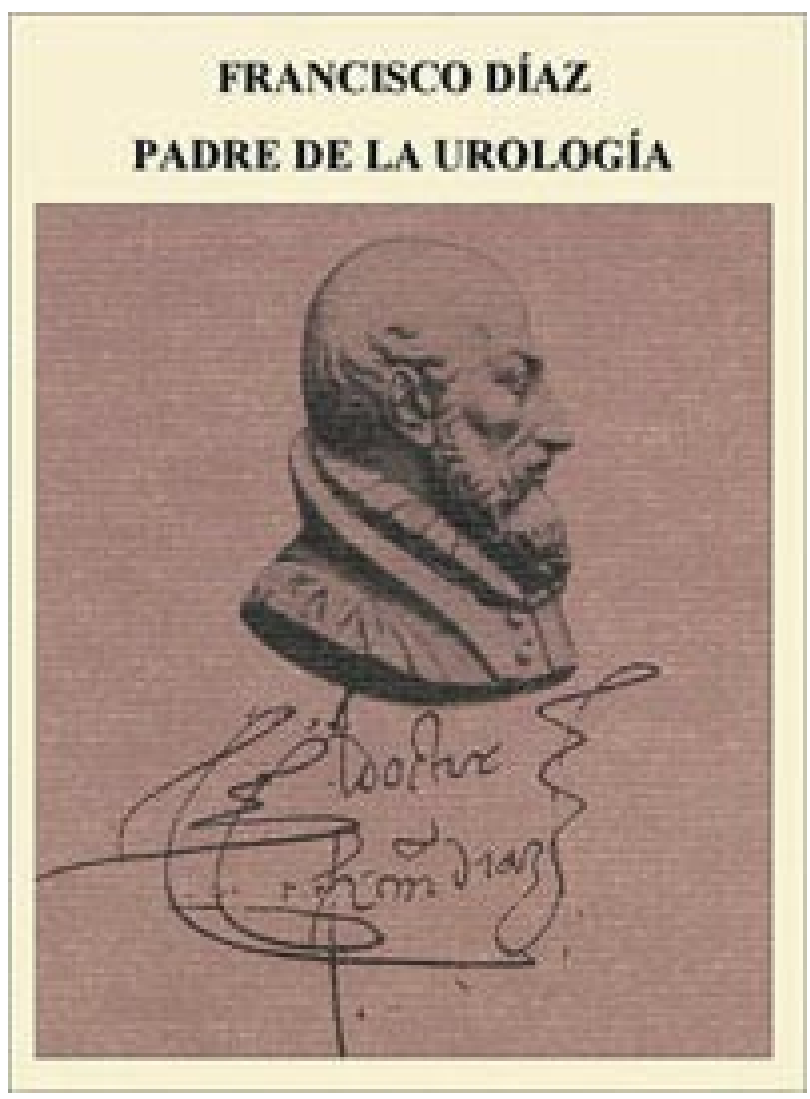

FIGURA 5. Francisco Díaz.
Storz. La modificación consistió en donde había aire poner una lente y donde antes había una lente poner aire. Este hecho revolucionó los endoscopios rígidos ya que además alargó la longitud de las lentes dando como resultado una imagen más brillante, más nítida y más grande.

La introducción de la fibra óptica en 1963 permitió mejorar la visión endoscópica mediante el uso de luz "fría", empleándose para el desarrollo de endoscopios flexibles.

El aprendizaje el aparato urinario inferior queda dominado por la endoscopia, o mejor dicho por la "Endourología". Un nuevo reto lo constituye el estudio y tratamiento de la patología ureteral y renal.

En 1941 se realiza la primera extracción endoscópica de un cálculo de un paciente con una nefrostomia a cielo abierto y en 1955 comienza a difundirse la nefrostomia percutánea como método diagnostico y de derivación permanente.

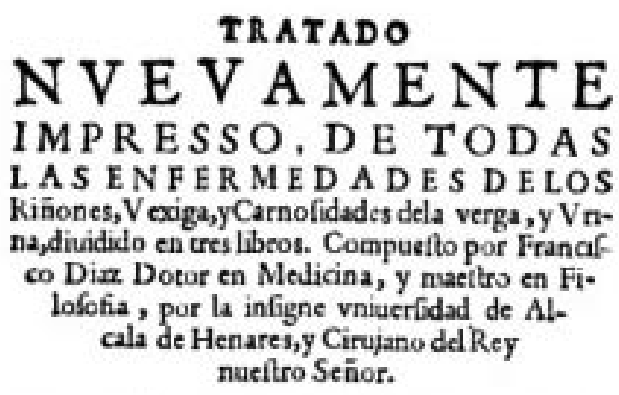

DIRIGIDO AL DOTOR VALLE

Protomedico del Rey nueftro Sarior,y Mrdico de fu Camara , \&c.

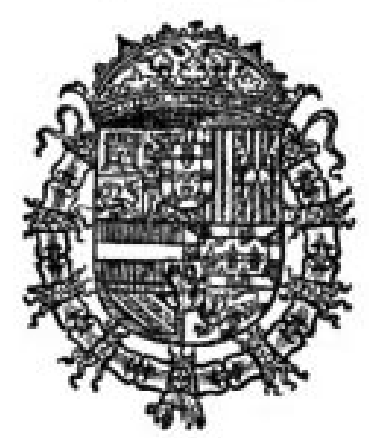

CON PRIVILEGIO.

Impreffo en Madrid por Francitco Sanchez. Año. 1588 .

FIGURA 6. "Tratado de las enfermedades de los riñones, vejiga y carnosidades de la verga y orina" de Francisco Díaz, publicado en 1588. 
Pasan unos años y en 1977 la Escuela de Maguncia destruye ultrasonicamente un cálculo coraliforme y estandariza la técnica, y Alken (4) en 1981, describe la dilatación del tracto de nefrostomia quedando el riñón al alcance del urólogo y del nefroscopio.

Es en 1979-1980 cuando en nuestro país Pérez Castro Ellendt (6) apoyado por su jefe de servicio el Dr Martínez Piñeiro, crea el primer ureterorrenoscopio lo que le permite el estudio del uréter.

De esta manera el perfeccionamiento del instrumental nos permite acceder en la actualidad endoscópicamente a cualquier punto del tracto urinario.

\section{TRATAMIENTO}

Hasta principios de la década de los ochenta toda litiasis urinaria se resolvía por cirugía abierta. Con el paso del tiempo el tratamiento de la misma ha sufrido un importante cambio debido al progreso de la tecnología.

El tratamiento de estos cálculos supone un reto para todo urólogo que debe estar entrenado en las diferentes técnicas. Dentro de ellas destacamos la cirugía abierta, la nefrolitotomia percutánea (NLP), la litotricia extracorpórea por ondas de choque (LEOC)

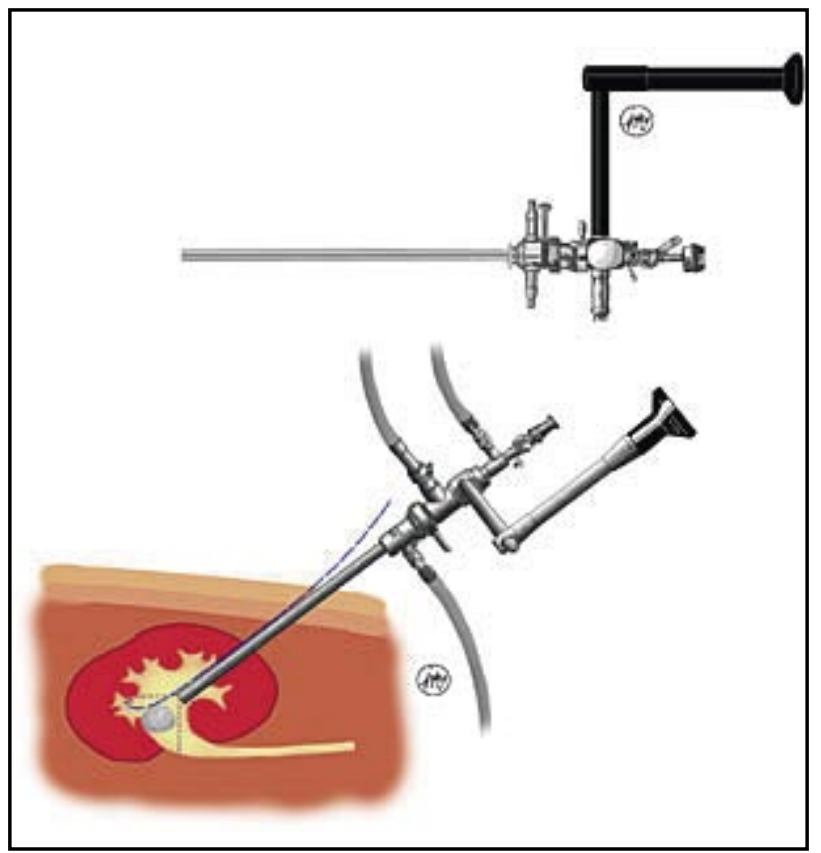

FIGURA 7. Nefroscopio, Técnica de nefrolitotomia percutánea. y la combinación de ésta última con la NLP ó la URS (ureterorrenoscopia).

Por su parte podemos también recurrir a tratamientos médicos como es el caso de la quimiolisis local a través de un catéter, utilizando bicarbonato - $\mathrm{N}$-acetilcisteina para las litiasis de ácido úrico o de cistina, y Renacidin o ácido cítrico en fragmentos litiasicos residuales de naturaleza infectiva, aunque tiene el inconveniente de producir toxicidad local y sistémica si se aplica durante largos periodos de tiempo.

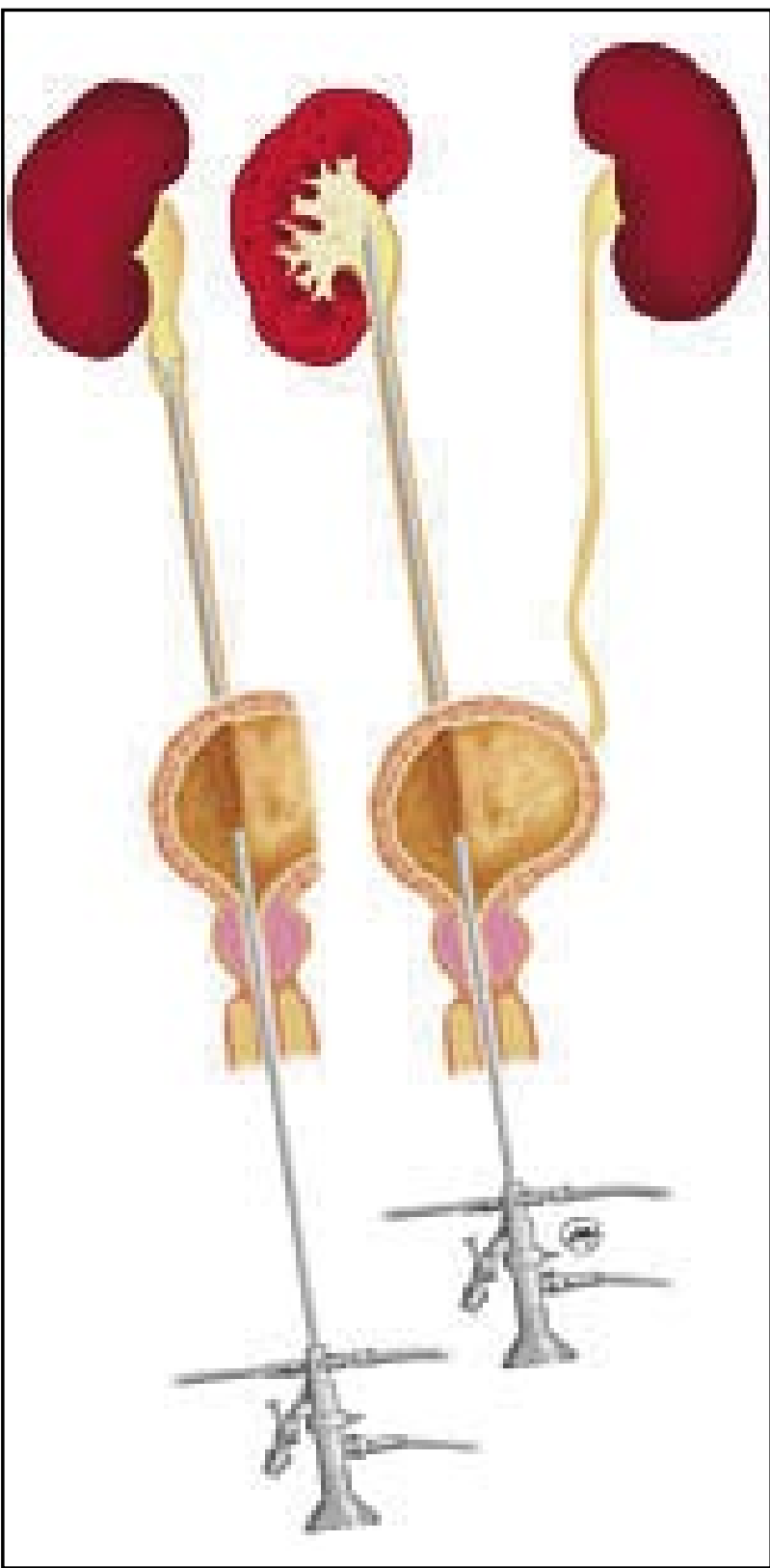

FIGURA 8. Ureterorrenoscopio. 
El creciente interés por el estudio de los factores médicos y la prevención de la litiasis propicio la creación de laboratorios especializados, siendo el pionero en nuestro país el de los doctores Cifuentes y Rapado, de la Fundación Jiménez Díaz de Madrid entre los años sesenta y setenta.

\section{CIRUGÍA ABIERTA}

Se considera que ha quedado reducida según las diferentes escuelas a un $4-6 \%$ de los casos de litiasis renal y que consistía en las mismas técnicas utilizadas antes de 1980, año en que aparece la endourología.

Estas técnicas son la pielotomía simple o intrasinusal, la nefrolitotomía anatrófica de Boyce, la pieloinfundibulotomía, la nefrectomía parcial, polar o total en caso de anulación funcional, y la ureterolitotomía en el caso de litiasis ureteral.

Si hacemos un poco de historia tenemos que remontarnos al siglo $\mathrm{XVI}$ para descubrir los primeros pasos de la cirugía renal. Se cree que la primera nefrolitotomía fue llevada a cabo en dos tiempos, por Doménico de Marchetti en 1860. Sin embargo las primeras nefrolitotomías regladas corresponden a las realizadas por Annandales en 1869 y por Ingalls en 1872.

A Sir Henry Morris $(4,7)$ se atribuye en 1884 la definición de los términos de nefrolitiasis, nefrotomia y nefrectomía.

El principal problema de estas intervenciones lo constituía las importantes perdidas hemáticas. Esto lleva a la búsqueda de una zona avascular intrarrenal la cual se conoce como nefrotomía anatrófica de Boyce, quien la describe en 1967.

Fundamental fue la aportación de J. $M^{a}$ Gil Vernet en 1960 de la pielotomia intrasinusal permitiendo la visualización de la porción intrasinusal de la pelvis y los cálices produciendo una mínima lesión del parénquima renal y sus vasos. Esto hizo que se constituyera en el procedimiento de elección para el tratamiento de la mayoría de los cálculos piélicos o combinada con nefrotomías radiales en el tratamiento de las litiasis coraliformes. Su trabajo fue presentado en el Congreso Internacional de Tokio en 1970.

Por su parte una solución drástica para el tratamiento de la litiasis renal es la nefrectomía que fue llevada a cabo por primera vez por Simon en 1869.

\section{CIRUGÍA ENDOUROLOGICA}

\section{Nefrolitotomía percutánea $(4,5)$}

La cirugía renal percutánea se consolida como técnica quirúrgica en 1981, cuando Alken y Wickham presentan el set telescopico de dilatación, el nefroscopio y el Litotritor ultrasónico que son los instrumentos fundamentales para realizar este acto quirúrgico.

Queda ya lejana su realización en dos tiempos, un primero para crear progresivamente el trayecto de nefrostomía y un segundo de litotricia propiamente dicho.

Es la fragmentación y extracción de litiasis tras la punción percutánea de la vía urinaria y la dilatación del canal de punción hasta que permita el paso del instrumento endourólogica de trabajo que es el nefroscopio.

En lo que respecta a la técnica el primer paso es seleccionar el cáliz más adecuado para el abordaje de las cavidades renales. Para ello es muy útil recordar un detalle anatomorradiológico estudiado por Wickham según el cual aproximadamente el $80 \%$ de los riñones tienen el "cáliz inferior" por debajo de la $12^{a}$ costilla, el cáliz medio un $36 \%$ y el cáliz superior un 18\%; he aquí por qué son los cálices inferiores la vía de acceso más común en la cirugía percutánea.

La posición del paciente es en decúbito prono o supino. A continuación opacifiamos la vía urinaria con contraste retrogradamente a través de un catéter ureteral colocado previamente. Acto seguido realizamos la punción percutánea de las cavidades renales a nivel del cáliz previamente seleccionado, dirigida bajo control ecográfico o radiológico. Se pasa una guía metálica flexible a través de la aguja de punción, dilatamos el trayecto percutáneo con dilatadores de teflón, balón o metálicos e instalamos la vaina de Amplatz y el nefroscopio (Figura 7). Posteriormente realizamos la litofragmentación y la extracción de los fragmentos litiasicos. La litofragmentación puede ser ultrasónica, electrohidraulica, por láser, neumática o electrocinética. Al finalizar la NLP es conveniente dejar una sonda de nefrostomia (SNP) durante $24 \mathrm{~h}$ y realizar un control radiológico con contraste antes de su retirada para comprobar la existencia de litiasis residual o de coágulos. En algunas ocasiones es necesario además la colocación de un catéter ureteral doble J.

Actualmente la indicación más frecuente de litotricia renal percutánea está representa por el cálculo coraliforme, ya sea como monoterapia o eliminando una porción importante de la masa litiásica 
(cirugía litorreductora). En 1986, G. Taylly en una serie de 300 casos concluía diciendo: "Probablemente no exista técnica más gratificante que la nefrolitotomía percutánea".

\section{Ureterorrenoscopia (6)}

Es una técnica que nos permite la visualización directa, endoscópica del aparato urinario superior por vía transuretral mediante la introducción del ureteroscopio, siendo la creación del mismo $100 \%$ español.

Es en 1979-1980 cuando en nuestro país Pérez-Castro Ellendt crea la ureterorrenoscopia (Figura 8) para realizar la técnica descrita. El primer modelo que se desarrolló era corto pero por ello fácil de manejar. Posteriormente se crean otros de mayor longitud que permitían llegar hasta el riñón. Al principio al no existir ningún sistema especial, las litiasis se atrapaban con sondas tipo Dormia. Tiempo después se crean las pinzas, primero de rama corta y luego de rama larga lo que facilitaba la extracción de los cálculos.

Posteriormente vinieron los diferentes tipos de energía como la electrohidraúlica, ultrasónica, hasta el láser de Holmio que hoy empleamos permitiendo la fragmentación sencilla de la litiasis.

\section{LITOTRICIA EXTRACORPOREA POR ONDAS DE CHOQUE (7)}

La posibilidad de destruir un cálculo de forma extracorpórea, es decir, sin afectar la superficie corporal del individuo proviene de las observaciones realizadas por los pilotos de los primeros aviones supersónicos. Se descubre que las ondas generadas bajo el agua eran capaces de atravesar los tejidos vivos sin daño demostrable. Los ingenieros de la firma aeronáutica Dornier fueron los primeros que tuvieron la idea de utilizar estas ondas de choque para la destrucción de los cálculos renales.

Es en 1980 cuando Chaussy en el Hospital Universitario de Munich realiza la primera litotricia en humanos. Después de su aprobación en 1984 por la FDA americana, en los años siguientes el método se introdujo en la mayoría de los hospitales del mundo para el tratamiento de la litiasis renal.

Su desarrollo es tan grande, pese a la juventud de los métodos, que hace que en 1982 se funde (4) la "Sociedad Mundial de Endourología y Ondas de choque" con sede en New York, existiendo material suficiente para celebrar una reunión anual.

\section{CONCLUSIONES}

Mediante esta revisión histórica se ha expuesto los comienzos del "Mal de la Piedra". Hemos visto como el desarrollo y el esfuerzo de muchos y grandes médicos han permitido no solo estudiar la formación de las litiasis, sino llevar a cabo su tratamiento comenzando por la cirugia abierta y siguiendo con el desarrollo de la endourología y de la litotricia por ondas de choque.

\section{BIBLIOGRAFÍA y LECTURAS RECOMENDADAS ( ${ }^{*}$ lectura de interés $y^{* *}$ lectura fundamental)}

*1. GMS Urología: Litiasis urinaria. Grupo Masson, Editorial Garsi, S.A. 1999: 5.

2. Sevilla Cecilia C, Pascual García X, Villavicencio Mavrich $\mathrm{H}$. Breve historia del tratamiento de la litiasis vesical. Actas Urol Esp, 2005; 29:10.

3. Ruíz Marcellán F J, Ibarz Servio L, Salinas Duffo D. Litiasis infecciosa. Tratamiento y complicaciones. Arch Esp Urol, 2001; 54: 937-950.

*4. Fernández del Busto E, Trueba Arguiñarena FJ. Endourología del Tracto Urinario Inferior. Secretariado de Publicaciones. Universidad de Valladolid, 1991; 9.

*5. J. Vicente R. Tratado de Endourología. Pulso ediciones s.a. Barcelona, 1996; 13

6. Pérez-Castro Ellendt E. Inicio y desarrollo de la ureterorrenoscopia. Arch.Esp.Urol. 2007; 60,8: 985-988.

7. Ruíz Marcellán F J, Ibarz Servio L. Historia de la litotricia por ondas de choque en España. Arch Esp Urol, 2007; 60, 8: 1003-1008. 